\title{
Analytical model for computing translational and rotational angular momentum occurring in treadmill walking
}

\author{
Dan Ioan Stoia ${ }^{1, *}$ and Alin-Florin Totorean ${ }^{1}$ \\ ${ }^{1}$ Department of Mechanics and Strength of Materials, Politehnica University of Timisoara, Romania
}

\begin{abstract}
The kinematical modifications of human gait associated with treadmill walking are well studied in the literature. Fewer researches are focusing on computing the dynamical parameters of the gait, in this particular situation. Starting from kinematical data recorded in treadmill walking, the paper proposes an analytical model of the lower limbs that allows computation of translational and rotational angular momentum for each segment. The experimental data used in the study were recorded using ultrasound based, 3D motion equipment. By mean of this system, relative and absolute angles of the lower limb can be computed using Cartesian coordinates of each anatomical landmark. The velocities and accelerations were obtained by numerical derivative. In order to compute the dynamical parameters, segment masses and inertias were collected from the literature. The masses are based on percentage of total body weight while the segment inertias are based on geometrical characteristics of lower limb segments.
\end{abstract}

\section{Introduction}

Instrumented treadmill walking is a non-invasive technique used in diagnosis and monitoring in patients with conditions like stroke [1], spinal cord injuries [2,3] or lower limb pathologies [4,5]. Therefore, instrumented treadmill-based gait analysis is a useful tool for monitoring and motion improvement in patient rehabilitation procedure. There have been performed numerous studies focused on measuring spatio-temporal, kinematics and kinetics parameters associated to human gait [6]. Results are highly influenced by the individual gait pattern, respectively the walking speed $[3,7]$. Our previous research was focused on measuring and computing kinematical parameters (absolute and relative angles) based on experimental data associated to healthy and pathological gait [5].

A main direction in the human motion analysis is to describe the dynamic parameters associated to human gait. There are studies showing that dynamic parameters such as the reaction forces and moments were measured based on treadmill belts equipped with force sensors $[8,10-14]$ but only few are focused on computing dynamical parameters of the gait, starting from the kinematical measured parameters [9].

${ }^{*}$ Corresponding author: dan.stoia@upt.ro 
Some other authors applied nonlinear analysis to the flexion-extension movements of the human knee both on healthy and osteoarthritic individuals, observing the increasing of Largest Lyapunov Exponents for the OA patients with respect to healthy subjects $[15,16]$.

The aim of this paper is to compute new parameters associated to human gait based on the experimental kinematical data. The kinetic parameters considered to be computed are the angular momentum and the translational momentum,

\section{Experimental setup}

The measurements were performed on a healthy subject, using Zebris Motion Analysis equipment from the Laboratory of Motion Analysis within the Politehnica University of Timisoara.

Using two emitters ultrasounds are produced and received by the markers positioned on the thigh and foot of the subject's lower limbs. The anatomical landmarks used in data recording are positioned according to the Figure 1. The recorded data represents $\mathrm{x}, \mathrm{y}$ and $\mathrm{z}$ coordinates of each landmark during the time of treadmill walking.

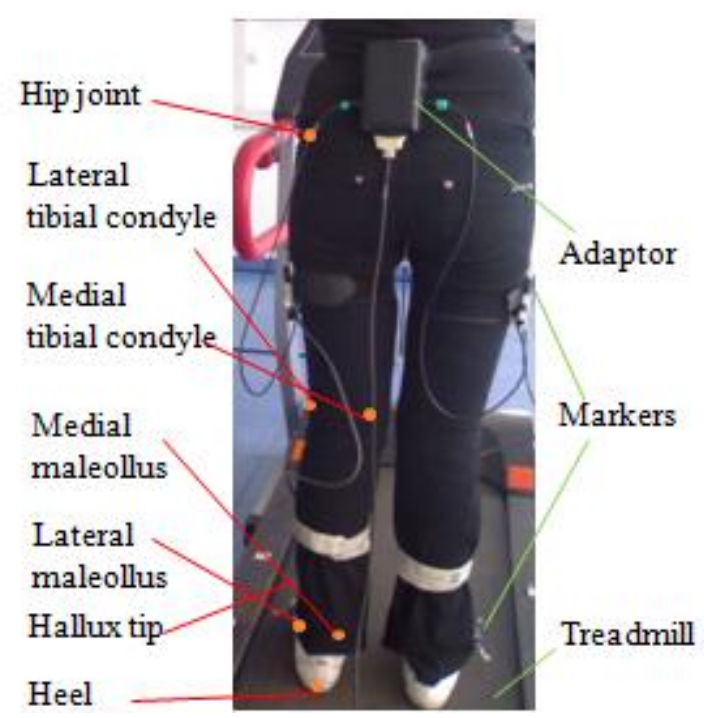

Fig. 1. Equipment setup and anatomical landmarkers of the subject.

Two different walking velocities of one and five $\mathrm{km} / \mathrm{h}$ were imposed on subject by mean of electrical treadmill. For each walking velocity 5 trials were recorded. At the end of the experiment, the time series of coordinates were averaged. Prior to recording trials, the subject spends 10 minutes of training in order to accommodate with the treadmill. The sampling rate of the recording system was set to $25 \mathrm{~Hz}$.

Coordinates of each landmarker were determined for a several consecutive motion cycles. The second step in kinematical part of the study was to compute the anatomical angles and the derivative parameters of these (by numerical derivation). By using the moment of inertia described in literature, rotational angular momentum was computed in relation to the segment's centre of mass $(\mathrm{CoM})$ and translational angular momentum was computed in relation to the foot centre of mass. Figure 2 shows the scheme with the steps followed in the new parameters computation. 


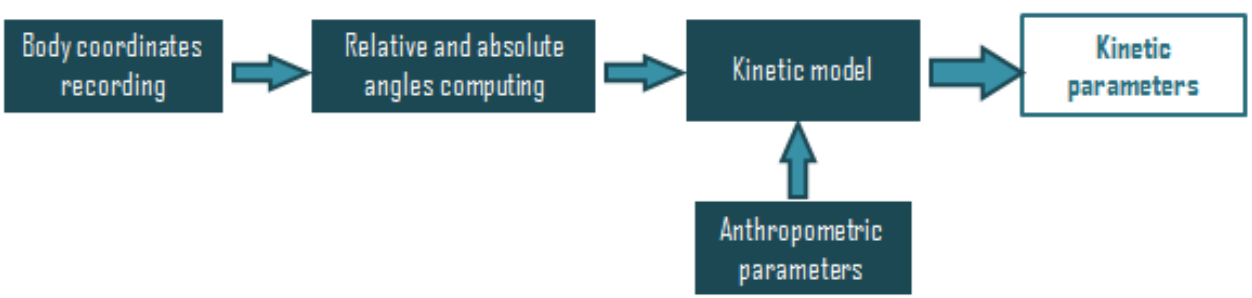

Fig. 2. The scheme for new parameter computation.

\section{Analytical approach}

Gait kinetics has to be approached according to the gait phases. This because in some sub phases the fixed point of the reference limb is on ground contact while on other sub phases the fixed point belongs to the contralateral limb.

The angular momentum of one segment according to a chosen point can be separated [9] in a sum of two independent components (Figure 3):

- orbital component $\left(\vec{K}_{o}\right)$ - computed using the velocity of the center of mass of the segment, its own mass, and the position vector.

- spin component $\left(\vec{K}_{s}\right)$ - computed considering the rotation axis of the segment passing through the center of mass of the segment.

The paper focuses on establishing the analytical model for computing shank's kinetic parameters, according to the foot. The model can be used for pre-swing phase of the gait. For any other gait phase or lower limb segment, the model should be readapted.

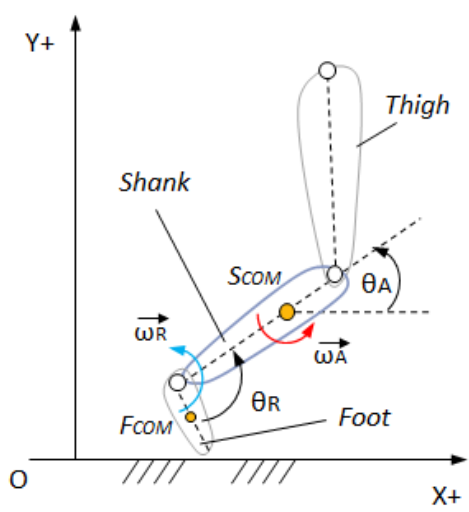

(a) Kinematical parameters considered in preswing phase;

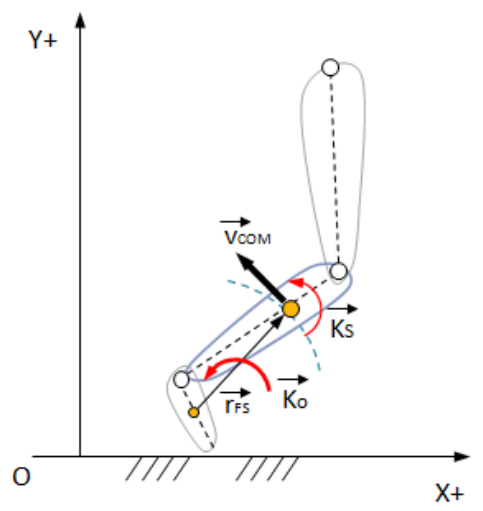

(b) Kinetic parameters considered in pre-swing phase;

Fig. 3. Kinematical and kinetic parameters representation for pre-swing phase.

The definitions of angular momentum components are:

$$
\begin{gathered}
\vec{K}_{o}=\vec{r}_{F S} \times\left(m_{S} \cdot \vec{v}_{C M}\right) \\
\vec{K}_{S}=J_{S} \cdot \vec{\omega}_{C M}
\end{gathered}
$$

Total angular momentum of the shank can be expressed as it follows: 


$$
\begin{gathered}
\vec{K}_{s e g}=\vec{K}_{o}+\vec{K}_{s} \\
\vec{K}_{s e g}=\vec{r}_{F S} \times\left(m_{s} \cdot \vec{v}_{C M}\right)+J_{s} \cdot \vec{\omega}_{C M} \\
J_{s}=m_{s} \cdot(\rho \cdot l)^{2} \\
\left\{\begin{array}{l}
x_{C M}=x_{p}+\% C M \cdot\left(x_{D}-x_{p}\right) \\
y_{C M}=y_{p}+\% C M \cdot\left(y_{D}-y_{p}\right)
\end{array}\right.
\end{gathered}
$$

Where the parameters can be described and defined as presented in Table 1:

Table 1. Parameters used for analytical modeling.

\begin{tabular}{|l|l|}
\hline Shank anthropometric parameters $[17,18]$ & Shank measured parameters \\
\hline $\begin{array}{l}m_{s} \text { Segment mass }[\mathrm{kg}] \text {, computed as \% of } \\
\text { the total body mass }\end{array}$ & $\vec{v}_{C M}-$ linear velocity of the center of mass \\
\hline $\begin{array}{l}\rho \text { Radii of gyration [m], computed as \% of } \\
\text { segment length }(l) \text { about a transverse axis }\end{array}$ & $\begin{array}{c}\vec{\omega}_{C M} \text { - angular velocity according to the } \\
\text { center of mass rotation axis }\end{array}$ \\
\cline { 2 - 3 } $\begin{array}{l}\text { \% CM - center of mass percentage } \\
\text { according to the segment }\end{array}$ & $\vec{J}_{S}$ - mass moment of inertia \\
\hline $\mathrm{X}_{\mathrm{CM}}$ and $\mathrm{Y}_{\mathrm{CM}}$ Center of mass coordinates & $\theta_{R}-$ foot-shank relative angle \\
\hline $\begin{array}{l}\left(\mathrm{X}_{\mathrm{p}}, \mathrm{Y}_{\mathrm{p}}\right) \text { and }\left(\mathrm{X}_{\mathrm{D}}, \mathrm{Y}_{\mathrm{D}}\right) \text { proximal and distal } \\
\text { coordinates of the segment }\end{array}$ & $\begin{array}{l}\theta_{A}-\text { absolute angle of the shank respecting } \\
\text { horizontal plane }\end{array}$ \\
\hline
\end{tabular}

\section{Results}

Two kinematical parameters recorded are presented in the figures 4 and 5. Here, the trajectories of the centre of mass for foot and shank segments are depicted for the two velocities. The loop shape of each is generated by the lack of progression for the body centre of mass, due to treadmill walking. The almost horizontal lines of the loops are representing stance phases while the $\mathrm{Y}$ coordinate elevations are belonging to the swing phases.

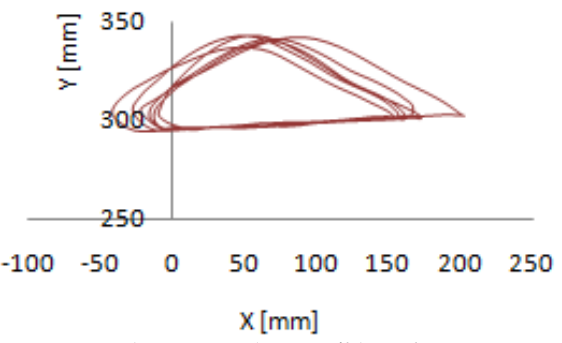

a) Low $(1 \mathrm{Km} / \mathrm{h})$ velocity;

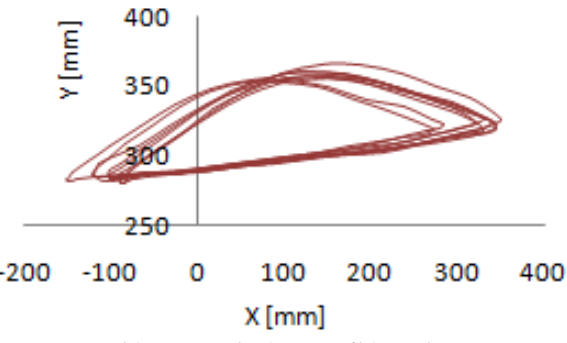

b) High $(5 \mathrm{Km} / \mathrm{h})$ velocity;

Fig. 4. Shank CoM trajectory. 


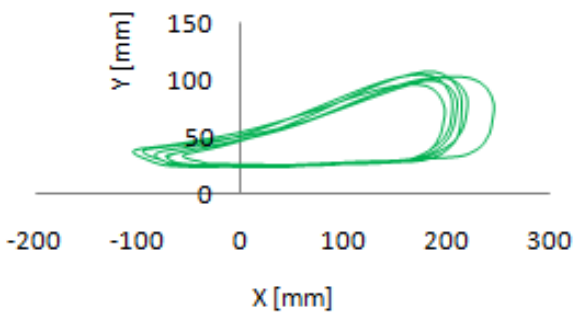

a) Low $(1 \mathrm{Km} / \mathrm{h})$ velocity;

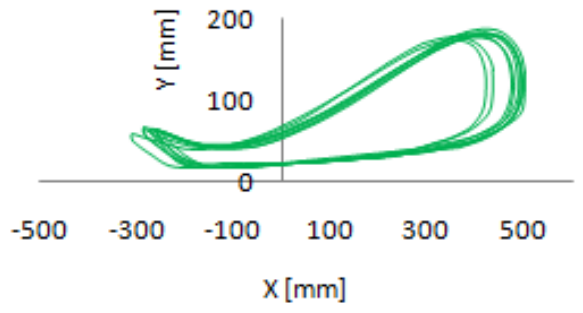

b) High $(5 \mathrm{Km} / \mathrm{h})$ velocity;

Fig. 5. Foot CoM trajectory.

Using the landmark coordinates the relative and absolute angles of the lower limb were computed. Based on these computations, the angular velocities and accelerations were obtained by numerical derivation. Because of the multitude of parameters that have to be determined prior to angular momentum, they are not presented in the paper.

Figure 6 shows the spin angular momentum of the shank corresponding to the investigated velocities. The low values for spin angular momentum are associated with low angular velocity around shank CoM and low moment of inertia of the shank, respecting the same axis. The frequencies of the signals are corresponding to the angular frequencies of movement.

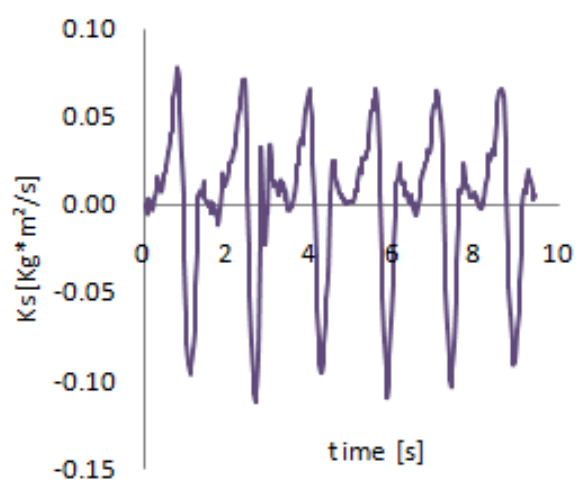

a) $\operatorname{Low}(1 \mathrm{Km} / \mathrm{h})$ velocity;

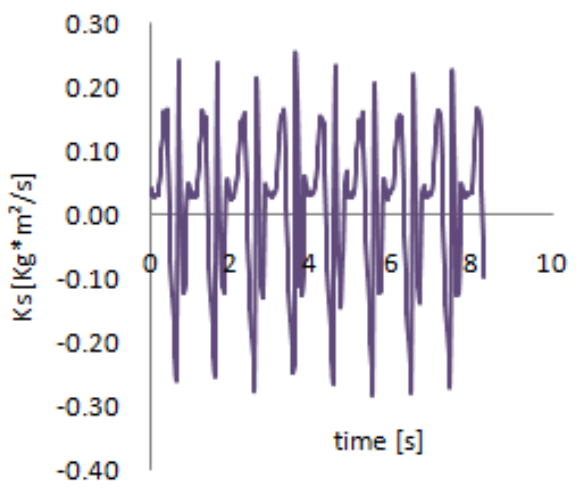

b) High $(5 \mathrm{Km} / \mathrm{h})$ velocity;

Fig. 6. Spin angular momentum of the shank.

The orbital momentums of the shank computed for the low and high velocities are presented in Figure 7. The computation of this parameter rely on equation 1 and was accomplished taking into consideration the foot $\mathrm{CoM}$ as reference. The values are around five times higher than those of spin angular momentum, mainly because of high linear velocity of the shank's CoM and large position vector between the two centers.

The total angular momentum of the shank segment was computed as sum of the spin and orbital angular momentums. Due to the time variation of the initial kinematical parameters, the kinetic parameters obtained are also functions of time. The summation of those was done according to the time interval and was possible due to the identical frequencies of the signals. The results are presented in the figure 8 , for the two linear velocities. It can be observed that increasing the linear velocity of the treadmill up to five $\mathrm{km} / \mathrm{h}$ the total angular momentum of the shank doubles. 


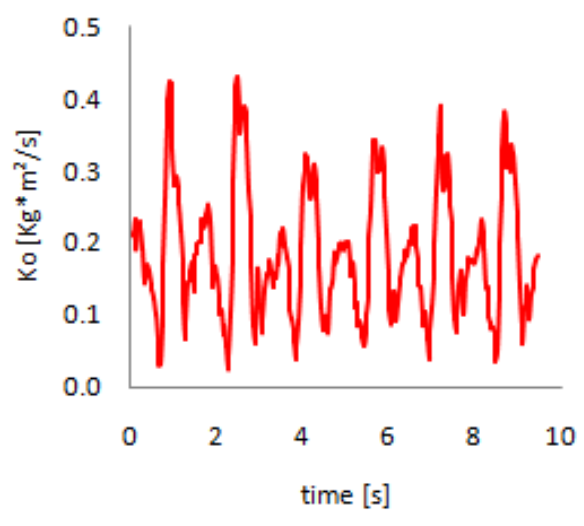

a) Low $(1 \mathrm{Km} / \mathrm{h})$ velocity;

Fig. 7. Orbital angular momentum of the shank.

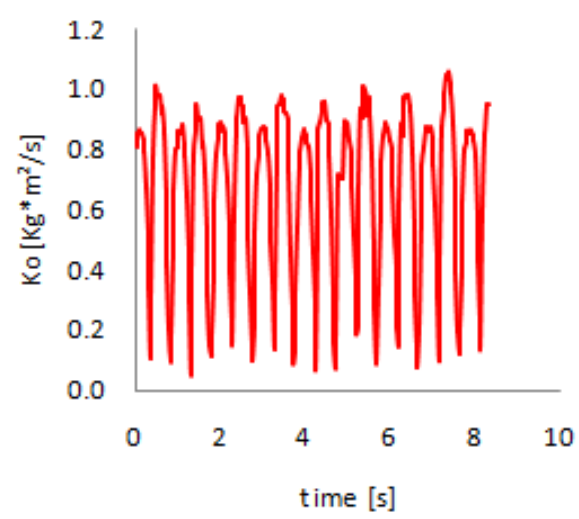

b) High $(5 \mathrm{Km} / \mathrm{h})$ velocity;
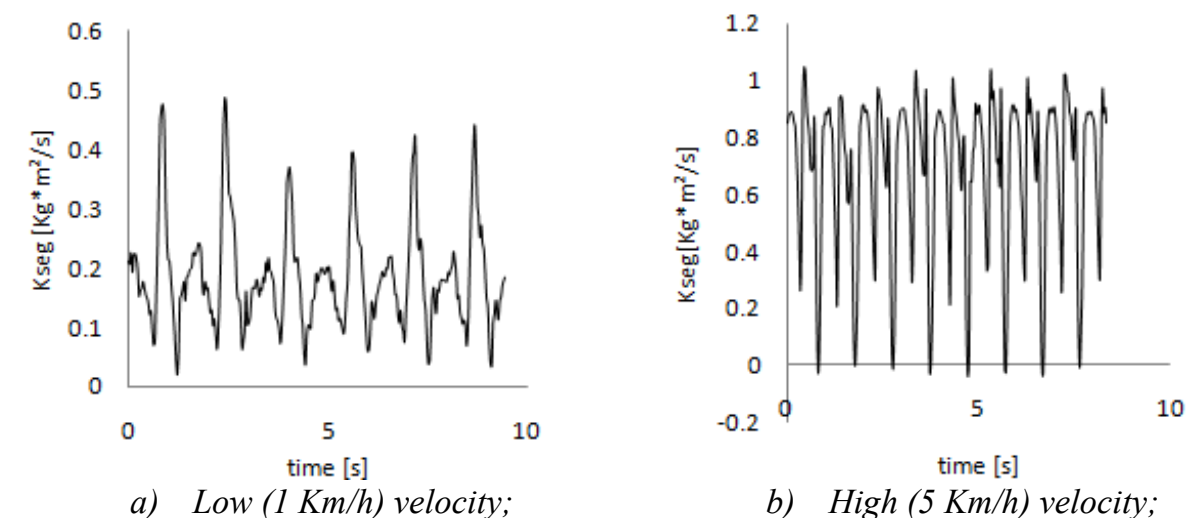

Fig. 8. Total angular momentum of the shank.

\section{Conclusions}

The treadmill walking presents some particularities with direct influence on spatio-temporal and kinematical parameters of the gait. This study offers some information regarding the kinematical parameters of the treadmill gait in close relation to the treadmill's velocity.

The analytical model permitted to obtain new parameters like angular momentum, computed using both experimental data and classical mechanics concepts. The computed data offer more complex information regarding the biomechanics of motion, and therefore the usefulness in clinical rehabilitation can be extend.

\section{References}

1. P.W. Duncan, K.J. Sullivan, A.L. Behrman, S.P. Azen, S.S. Wu, S.E. Nadeau, B.H. Dobkin, D.K. Rose, J.K. Tilson, S.Cen, S.K. Hayden, Body-weight-supported treadmill rehabilitation after stroke, $N$ Eng $J$ Med 2011; 364:2026-2036 (2011)

2. M. Wu, J.M. Landry, B.D. Schmit, T.G. Hornby, S.-C. Yen, Robotic resistance treadmill training improves locomotor function in human spinal cord injury: a pilot study, Archives of Physical Medicine and Rehabilitation, 93(5), $782-789$ (2012). 
3. A. Pepin, K.E. Norman, H. Barbeau, Treadmill walking in incomplete spinal-cordinjured subjects: 1. Adaption to changes in speed, Spinal Cord, 41, 257-270 (2003).

4. H. Houdijk, M.W. van Ooijen, J.J. Kraal, H.O. Wiggerts, W. Polomski, T.W.J. Janssen, M. Roerdink, Assessing Gait Adaptability in People With a Unilateral Amputation on an Instrumented Treadmill With a Projected Visual Context, Physical Therapy, 92(11), 1452-1460 (2012).

5. D.I. Stoia, C. Vigaru, L. Rusu, Relative and absolute angles computed from pathologic gait data, Springer Proceedings in Physics, 198, 201-206 (2018).

6. C.A. Fukuchi, R.K. Fukuchi, M. Duarte, A public dataset of overground and treadmill walking kinematics and kinetics in healthy individuals, PeerJ, 6, e4640 (2018).

7. A.J.J. Smith, E.D. Lemaire, Temporal-spatial gait parameter models of very slow walking, Gait\&Posture, 61, 125-129 (2018).

8. L.H. Sloot, M.M. van der Krogt, J. Harlaar, Self-paced versus fixed speed treadmill walking, 39, 478-484 (2014).

9. B.M.M. Gaffney, C.L. Christiansen, A.M. Murray, A.K. Silverman, B.S. Davidson, Separation of rotational and translational segmental momentum to assess movement coordination during walking, Human Movement Science, 51, 99-111 (2017).

10. J Li, S Wang, Q Zhang, C Tao, R Ji , Effects of Treadmill Speed on Joint Angles of Human Lower Limbs During Walking, Computer Science and Engineering Technology (CSET2015), Medical Science and Biological Engineering (MSBE2015).

11. S. Focke Martinez ; O. Kuzmicheva ; A. Gräser, Prediction of characteristic points of hip and knee joint trajectories during overground walking using IMUs and Artificial Neural Networks, 2016 IEEE International Symposium on Medical Measurements and Applications (MeMeA), (2016).

12. Sun, J., Wang, Y., Li, J., Wan,W., Cheng, D., Zhang, H.,View-invariant gait recognition based on kinect skeleton feature, Multimed Tools Appl, 77(295) (2018).

13. D Tarnita, M Catana, DN Tarnita, Nonlinear analysis of normal human gait for different activities with application to bipedal locomotion, Rom. J. Techn. Sci. - Appl. Mechanics., 58(1-2), 177-192 (2013).

14. R. Luca , Statistical analysis of some parameters describing human locomotion, Proceedings of 5th IEEE International Conference on E-Health and Bioengineering, 2015

15. Tarnita, D., D-B Marghitu, Nonlinear dynamics of normal and osteoarthritic human knee, Proceedings of the Romanian Academy, pp. 353-360, 2017.

16. Tarnita, D., Georgescu, M., Tarnita, D.N., Applications of Nonlinear Dynamics to Gait Analysis on Plane\& Inclined Treadmill, New Trends in Medical and Service Robots, Springer Publishing House, Vol 39, 59-73, 2016.

17. J. Hamill, K.M. Knutzen, Biomechanical Basics of Human Movement, Second edition, Lippincott Williams \&Wilkins, 2003.

18. Dempster, W. T. (Wilfrid Taylor), Space requirements of the seated operator: geometrical, kinematic, and mechanical aspects of the body, with special reference to the limbs, 1955 